\title{
Correction to: Predictors of localization, outcome, and etiology of spontaneous intracerebral hemorrhages: focus on cerebral amyloid angiopathy
}

\author{
Bernadett Fakan $^{1} \cdot$ Zita Reisz $^{2} \cdot$ Denes Zadori $^{1} \cdot$ Laszlo Vecsei $^{1,3} \cdot$ Peter Klivenyi $^{1} \cdot$ Levente Szalardy $^{1}$ (D)
}

Published online: 9 May 2020

(c) The Author(s) 2020

\section{Correction to: Journal of Neural Transmission https://doi.org/10.1007/s00702-020-02174-2}

The original version of this article unfortunately contained a mistake. Mistake in Tables 1 and 2.

Indeed, the Tables 1 and 2 has a superscript "a" as footnote of variables with significant difference in the multivariate model. This superscript "a" originally was before the measure in the bracket (i.e. (y) or (\%)), as approved by the authors in the proof. However, the superscript "a" was not before but after the measure in the bracket yielding e.g. (y) a, in the first published version of the manuscript in Tables 1 and 2.

However, this change in fact resulted in a typo in Table 1, namely "Age at eventa" with an attached "a" after "event" in the same font size.

The corrected Tables 1 and 2 are placed in the following page.

The original article has been corrected.
The original article can be found online at https://doi.org/10.1007/ s00702-020-02174-2.

Levente Szalardy

szalardy.levente@med.u-szeged.hu

1 Department of Neurology, Faculty of Medicine, Albert Szent-Györgyi Clinical Center, University of Szeged, Semmelweis u. 6, 6725 Szeged, Hungary

2 Department of Pathology, Faculty of Medicine, Albert Szent-Györgyi Clinical Center, University of Szeged, Állomás u. 2, 6725 Szeged, Hungary

3 MTA-SZTE Neuroscience Research Group, Semmelweis u. 6, 6725 Szeged, Hungary 
Table 1 Discriminators of spontaneous ICHs with regard to localization

\begin{tabular}{|c|c|c|c|c|c|}
\hline & \multirow{2}{*}{$\begin{array}{l}\text { Lobar/cerebellar } \\
\mathrm{ICH}\end{array}$} & \multirow{2}{*}{$\begin{array}{l}\text { Deep } \\
\text { ICH }\end{array}$} & \multirow{2}{*}{$\begin{array}{l}{\mathrm{MW} / \mathrm{Chi}^{2}}^{2} \\
p\end{array}$} & \multicolumn{2}{|c|}{ multivariate logistic regression } \\
\hline & & & & $p$ & OR $(95 \% \mathrm{CI})$ \\
\hline Patient number & 92 & 121 & - & - & \\
\hline Aage at event ${ }^{\mathrm{a}}(\mathrm{y})$ & $74.5[65.9-82.0]$ & $64.7[57.9-76.6]$ & $<0.001$ & 0.014 & $1.03(1.01-1.06)$ \\
\hline Sex (male/female) (\%) & 52.2 & 66.9 & 0.029 & $>0.05$ & - \\
\hline Prior ischemic stroke (\%) & 12.0 & 12.7 & $>0.05$ & - & - \\
\hline Prior intracranial hemorrhage $(\%)$ & 7.7 & 8.5 & $>0.05$ & - & - \\
\hline Prior TIA (TFNE) $(\%)$ & 14.1 & 7.6 & $>0.05$ & - & - \\
\hline Prior loss of consciousness $(\%)$ & 9.8 & 6.8 & $>0.05$ & - & - \\
\hline Family history for any stroke $(\%)$ & 37.5 & 28.8 & $>0.05$ & - & - \\
\hline Anticoagulant use (\%) & 20.9 & 13.4 & $>0.05$ & - & - \\
\hline INR > $1.4(\%)$ & 18.4 & 11.4 & $>0.05$ & - & - \\
\hline Antiplatelet use ${ }^{\text {a }}(\%)$ & 43.3 & 23.7 & 0.003 & 0.043 & $1.96(1.02-3.75)$ \\
\hline Combined antithrombotic use (\%) & 13.3 & 3.4 & 0.016 & $>0.05$ & - \\
\hline Hypertensive excess $^{\mathrm{a}}(\%)$ & 48.9 & 71.2 & 0.001 & 0.002 & $0.39(0.21-0.71)$ \\
\hline Chronic hypertension (\%) & 88.0 & 90.9 & $>0.05$ & - & - \\
\hline Case fatality (1-month) (\%) & 34.8 & 33.1 & $>0.05$ & - & - \\
\hline
\end{tabular}

$\mathrm{MW} / \mathrm{Chi}^{2}$, Mann-Whitney test (for Age at event) or $\mathrm{Chi}^{2}$ test (for other variables), $C I$ confidence interval, $I C H$ intracerebral hemorrhage, $I N R$ international normalized ratio, OR odds ratio, TIA transient ischemic attack, TFNE transient focal neurological episode, $y$ year (median [interquartile range])

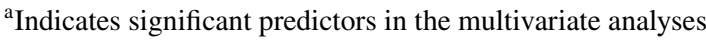

Bold font indicates variables with significant difference in univariate analyses

Table 2 Discriminators of spontaneous ICHs with regard to probable/definite CAA diagnosis

\begin{tabular}{|c|c|c|c|c|c|}
\hline & \multirow{2}{*}{$\begin{array}{l}\text { Probable/definite } \\
\text { CAA }\end{array}$} & \multirow{2}{*}{$\begin{array}{l}\text { Non-probable } \\
\text { CAA }\end{array}$} & \multirow{2}{*}{$\begin{array}{l}\mathrm{St} / \mathrm{Chi}^{2} \\
p\end{array}$} & \multicolumn{2}{|c|}{ multivariate logistic regression } \\
\hline & & & & $p$ & OR $(95 \% \mathrm{CI})$ \\
\hline Patient number & 16 & 152 & - & - & \\
\hline Age at event ${ }^{\mathrm{a}}(\mathrm{y})$ & $75.9 \pm 2.3$ & $65.6 \pm 1.1$ & 0.002 & 0.012 & $1.08(1.02-1.15)$ \\
\hline Sex (male/female) (\%) & 37.5 & 64.5 & 0.035 & $>0.05$ & - \\
\hline Prior ischemic stroke $(\%)$ & 18.8 & 10.7 & $>0.05$ & - & - \\
\hline Prior intracranial hemorrhage $^{a}(\%)$ & 31.3 & 6.8 & 0.008 & 0.005 & $8.53(1.94-37.58)$ \\
\hline Prior TIA (TFNE) (\%) & 31.3 & 7.4 & 0.010 & $>0.05$ & - \\
\hline Prior loss of consciousness $(\%)$ & 18.8 & 6.0 & $>0.05$ & - & - \\
\hline Family history for any stroke & 42.9 & 29.1 & $>0.05$ & - & - \\
\hline Anticoagulant use (\%) & 18.8 & 12.8 & $>0.05$ & - & - \\
\hline INR $>1.4(\%)$ & 20.0 & 10.4 & $>0.05$ & - & - \\
\hline Antiplatelet use $^{\mathrm{a}}(\%)$ & 56.3 & 25.2 & 0.009 & 0.042 & $3.45(1.05-11.38)$ \\
\hline Combined antithrombotic use (\%) & 6.4 & 4.1 & $>0.05$ & - & - \\
\hline Hypertensive excess (\%) & 46.7 & 64.2 & $>0.05$ & - & - \\
\hline Chronic hypertension (\%) & 93.8 & 88.8 & $>0.05$ & - & - \\
\hline Case fatality (1-month) $(\%)$ & 31.3 & 28.9 & $>0.05$ & - & - \\
\hline
\end{tabular}

$\mathrm{St} / \mathrm{Chi}^{2}$, Student $t$ test (for Age at event) or $\mathrm{Chi}^{2}$ test (for other variables); $C I$ confidence interval, ICH intracerebral hemorrhage, INR international normalized ratio, OR odds ratio, TIA transient ischemic attack, TFNE transient focal neurological episode, $y$ year (mean \pm SEM)

${ }^{a}$ Indicates significant predictors in multivariate analyses

Bold font: indicates variables with significant difference in univariate analyses 
Open Access This article is licensed under a Creative Commons Attribution 4.0 International License, which permits use, sharing, adaptation, distribution and reproduction in any medium or format, as long as you give appropriate credit to the original author(s) and the source, provide a link to the Creative Commons licence, and indicate if changes were made. The images or other third party material in this article are included in the article's Creative Commons licence, unless indicated otherwise in a credit line to the material. If material is not included in the article's Creative Commons licence and your intended use is not permitted by statutory regulation or exceeds the permitted use, you will need to obtain permission directly from the copyright holder. To view a copy of this licence, visit http://creativecommons.org/licenses/by/4.0/.

Publisher's Note Springer Nature remains neutral with regard to jurisdictional claims in published maps and institutional affiliations. 